\title{
Deeply Virtual Neutrino Scattering at Leading Twist*
}

\author{
C. Corianò ${ }^{\mathrm{a}} \mathrm{M}$. Guzzi ${ }^{\mathrm{b}}$ \\ ${ }^{a}$ Dipartimento di Fisica, Università di Lecce, and INFN sez. Lecce, \\ via per Arnesano, 73100 Lecce, Italy. \\ ${ }^{\mathrm{b}}$ Dipartimento di Fisica, Università di Lecce \\ via per Arnesano, 73100 Lecce, Italy.
}

We illustrate the generalization of results concerning exclusive electromagnetic processes in the deeply virtual limit (DVCS) to the case of the weak interactions. We briefly describe the derivation of the differential cross section for neutrino-nucleon reactions mediated by the neutral current and the amplitude for similar reactions mediated by the charged currents.

\section{Introduction}

Exclusive processes mediated by the weak force are an area of investigation which will gather, we believe, a wide interest in the forthcoming years due to the various experimental proposals to detect neutrino oscillations at intermediate energy using neutrino factories and superbeams, 1. Also, neutrino scattering on nucleons in the deeply virtual kinematics is important in order to draw a better picture of the hadronic interactions mediated by the weak currents. This is one of the simplest processes, among all, described by the Generalized Parton distribution functions (GPDFs) which unify the parton model description of both inclusive ed exclusive processes [2]3. Exclusive processes in the region of small momentum transfer (few $\mathrm{GeVs}$ ) are well described by hadronic wave functions - also called "distribution amplitudes" - using the ERBL formalism of Efremov-Radyushkin-Brodsky-Lepage (see 44 [5] for an overview), while inclusive processes at high energy are described by ordinary parton distributions. GPDs are the appropriate tool to describe the so called "Generalized Bjorken region" which is the region where, beside Bjorken's x variable, additional scaling variables appear. Nonforward parton distributions are the simplest constructs

*Presented at Neutrino Oscillations Workshop (NOW 2006), Otranto, Lecce, Italy, September 9-16 2006 belonging to this class and describe true lightcone correlators in the nucleon state, not built by unitarity as ordinary parton distributions, since the optical theorem does not hold. An asymmetry scaling parameter, in this case, $\xi$, defined below, characterizes the longitudinal momentum exchange between the initial and final momenta of the nucleon. The study of the electromagnetic interaction in this kinematical region, the Deeply Virtual Compton Scattering (DVCS) region, by now amounts to several hundreds of papers written in the last few years. Our objective, in this contribution, is to briefly outline the extension to the electroweak sector of these findings.

\section{DVNS kinematics}

A pictorial description of the process we are going to illustrate is given in Fig 1 where a neutrino of momentum $l$ scatters off a nucleon of momentum $P_{1}$ by an interaction with a neutral current; from the final state a photon and a nucleon emerge, of momenta $q_{2}$ and $P_{2}$ respectively, while the momenta of the final lepton is $l^{\prime}$

$P_{1,2}=\bar{P} \pm \frac{\Delta}{2} \quad q_{1,2}=\bar{q} \mp \frac{\Delta}{2}$,

with $-\Delta=P_{2}-P_{1}$ being the momentum transfer. Clearly

$\bar{P} \cdot \Delta=0, \quad t=\Delta^{2} \quad \bar{P}^{2}=M^{2}-\frac{t}{4}$ 


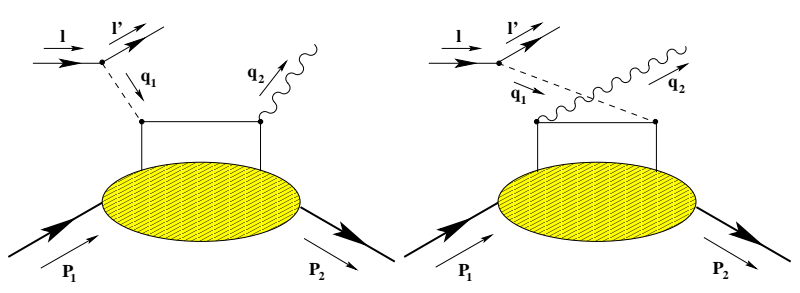

Figure 1. Leading hand-bag diagrams for the process

and $M$ is the nucleon mass. There are two scaling variables which are identified in the process, since 3 scalar products can grow large in the generalized Bjorken limit: $\bar{q}^{2}, \Delta \cdot q, \bar{P} \cdot \bar{q}$.

The momentum transfer $t=\Delta^{2}$ is a small parameter in the process. Momentum asymmetries between the initial and the final state nucleon are measured by two scaling parameters, $\xi$ and $\eta$, related to ratios of the former invariants

$\xi=-\frac{\bar{q}^{2}}{2 \bar{P} \cdot \bar{q}} \quad \eta=\frac{\Delta \cdot \bar{q}}{2 \bar{P} \cdot \bar{q}}$,

where $\xi$ is a variable of Bjorken type, expressed in terms of average momenta rather than nucleon and Z-boson momenta. The standard Bjorken variable $x=-q_{1}^{2} /\left(2 P_{1} \cdot q_{1}\right)$ is trivially related to $\xi$ in the $t=0$ limit. In the DIS limit $\left(P_{1}=P_{2}\right)$ $\eta=0$ and $x=\xi$, while in the DVCS limit $\eta=\xi$ and $x=2 \xi /(1+\xi)$, as one can easily deduce from the relations

$q_{1}^{2}=\left(1+\frac{\eta}{\xi}\right) \bar{q}^{2}+\frac{t}{4}, \quad q_{2}^{2}=\left(1-\frac{\eta}{\xi}\right) \bar{q}^{2}+\frac{t}{4}$.

We introduce also the inelasticity parameter $y=$ $P_{1} \cdot l /\left(P_{1} \cdot q_{1}\right)$ which measures the fraction of the total energy that is transferred to the final state photon.

\section{The Amplitudes}

The Compton amplitude is described by the following correlator involving neutral and charged currents

$T_{\mu \nu}=i \int d^{4} x e^{i q x}\left\langle P_{2}\left|T\left(J_{\nu}^{\gamma}(x / 2) J_{\mu}^{W^{ \pm}, Z_{0}}(-x / 2)\right)\right| P_{1}\right\rangle$.
The parameterizations of the non-forward light cone correlators in terms of GPD's is of the form given by Ji at leading twist [2]

$$
\begin{gathered}
\int \frac{d \lambda}{(2 \pi)} e^{i \lambda z}\left\langle P^{\prime}\left|\bar{\psi}\left(-\frac{\lambda n}{2}\right) \gamma^{\mu} \psi\left(\frac{\lambda n}{2}\right)\right| P\right\rangle= \\
H\left(z, \xi, \Delta^{2}\right) \bar{U}\left(P^{\prime}\right) \gamma^{\mu} U(P) \\
\quad+E\left(z, \xi, \Delta^{2}\right) \bar{U}\left(P^{\prime}\right) \frac{i \sigma^{\mu \nu} \Delta_{\nu}}{2 M} U(P)+\ldots . \\
\int \frac{d \lambda}{(2 \pi)} e^{i \lambda z}\left\langle P^{\prime}\left|\bar{\psi}\left(-\frac{\lambda n}{2}\right) \gamma^{\mu} \gamma^{5} \psi\left(\frac{\lambda n}{2}\right)\right| P\right\rangle= \\
\tilde{H}\left(z, \xi, \Delta^{2}\right) \bar{U}\left(P^{\prime}\right) \gamma^{\mu} \gamma^{5} U(P) \\
+\tilde{E}\left(z, \xi, \Delta^{2}\right) \bar{U}\left(P^{\prime}\right) \frac{\gamma^{5} \Delta^{\mu}}{2 M} U(P)+\ldots .
\end{gathered}
$$

which have been expanded in terms of functions $H, E, \tilde{H}, \tilde{E}$ which are the GPDs and the ellipses are meant to denote the higher-twist contributions. It is interesting to observe that the amplitude is still described by the same light-cone correlators as in the electromagnetic case (vector, axial vector) but now parity is not conserved.

In our calculations we use a simplified model for the GPD's where the $\Delta^{2}$ dependence can be factorized as follows 6 for a generic GPD

$H^{i}\left(z, \xi, \Delta^{2}, Q^{2}\right)=F_{1}^{i}\left(\Delta^{2}\right) q^{i}\left(z, \xi, Q^{2}\right)$,

where $F_{1}^{i}\left(\Delta^{2}\right)$ is a form factor, and the construction of the input distributions, in correspondence of an input scale $Q_{0}$, is performed using

$$
\begin{aligned}
& q\left(z, \xi, Q^{2}\right)=\int_{-1}^{1} d x^{\prime} \int_{-1+\left|x^{\prime}\right|}^{1-\left|x^{\prime}\right|} d y^{\prime} \delta\left(x^{\prime}+\xi y^{\prime}-z\right) \\
& \times f\left(y^{\prime}, x^{\prime}, Q^{2}\right) .
\end{aligned}
$$

As an example we show a plot of some GPDs in Fig (2), for the up and the down quarks as a function of the variable $z$ (a generalization of Bjorken-x, with $-1<z<1$ ) and at a fixed value of the variables $\Delta$ and $\xi$.

\section{The cross sections}

One possible differential cross section to study, in analogy to the DVCS case, is the following

$$
\frac{d \sigma}{d x d Q^{2} d\left|\Delta^{2}\right| d \phi_{r}}=\frac{y}{Q^{2}} \frac{d \sigma}{d x d y d\left|\Delta^{2}\right| d \phi_{r}}=
$$




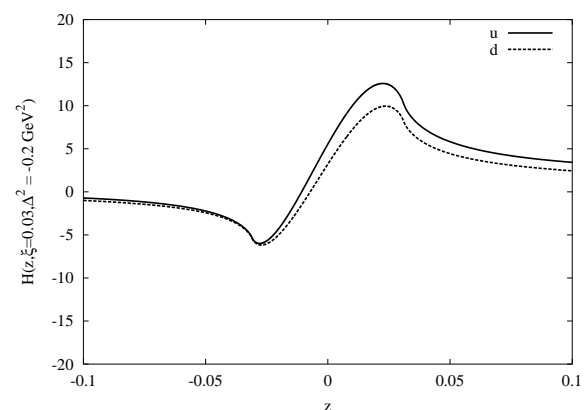

Figure 2. GPD's $H_{u}$ and $H_{d}$ generated by the diagonal parton distribution at an initial $0.26 \mathrm{GeV}^{2}$

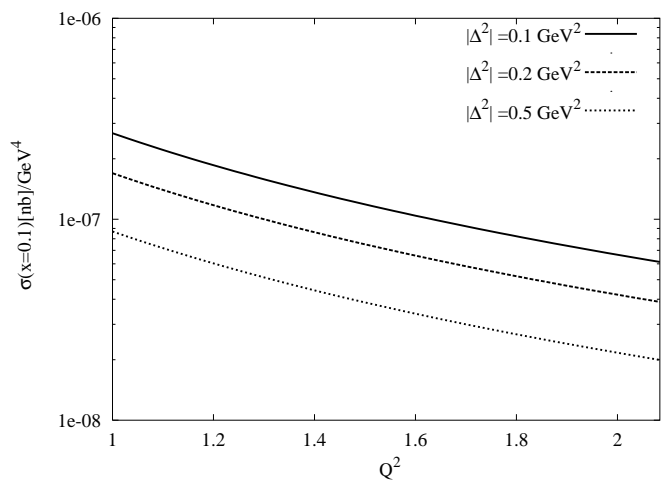

Figure 3. DVCS cross section at $x=0.1$ and center of mass energy $M E=10 \mathrm{GeV}^{2}$.

$$
\frac{x y^{2}}{8 \pi Q^{4}}\left(1+\frac{4 M^{2} x^{2}}{Q^{2}}\right)^{-\frac{1}{2}}\left|\mathcal{M}_{f i}\right|^{2},
$$

where $\phi_{r}$ is the angle between the lepton and the hadron scattering planes. $\left|\mathcal{M}_{f i}\right|^{2}$, as a functions of the invariants of the process, can be found in 7.

\section{Amplitudes in the charged sector}

We have also analized the expressions for the correlators of the charged electroweak current and derived their expressions in terms of the GDPs 8$]$.
These are formally given by the correlators

$$
\begin{gathered}
T_{\mu \nu}^{W^{+}}=i \int d^{4} x \frac{e^{i q x} x^{\alpha} U_{u d}}{2 \pi^{2}\left(x^{2}-i \varepsilon\right)^{2}}\left\langle P_{2}\right|\left[i S_{\mu \alpha \nu \beta}\left(\tilde{O}_{u d}^{\beta}+O_{u d}^{5 \beta}\right)\right. \\
\left.+\varepsilon_{\mu \alpha \nu \beta}\left(O_{u d}^{\beta}+\tilde{O}_{u d}^{5 \beta}\right)\right]\left|P_{1}\right\rangle, \\
T_{\mu \nu}^{W^{-}}=i \int d^{4} x \frac{e^{i q x} x^{\alpha} U_{d u}}{2 \pi^{2}\left(x^{2}-i \varepsilon\right)^{2}}\left\langle P_{2}\right|\left[-i S_{\mu \alpha \nu \beta}\left(\tilde{O}_{d u}^{\beta}+O_{d u}^{5 \beta}\right)\right. \\
\left.-\varepsilon_{\mu \alpha \nu \beta}\left(O_{d u}^{\beta}+\tilde{O}_{d u}^{5 \beta}\right)\right]\left|P_{1}\right\rangle,
\end{gathered}
$$

where the partonic content is extracted from the $O_{d u}$ operators while $S_{\mu \alpha \nu \beta}$ denote suitable combinations of Dirac gamma matrices. We refer to 8 for more details. We have suppressed the $x$ dependence of the operators in the former equations. The identification of the relevant partonic description in terms of GPDs has been obtained by us 8 using the leading-twist decomposition worked out in [9. Related work, using a different approach, can be found also in $[10$.

\section{Conclusions}

We have presented an extension of the standard DVCS process to the case of one neutral current exchange, describing the scattering of a neutrino off a proton in the parton model. We have described the leading twist behaviour of the cross section; we have found that this is comparable to other typical neutrino cross sections. The process is the natural generalization of DIS with neutral currents and relies on the notion of Generalized Parton Distributions, new constructs in the parton model which have received considerable attention in recent years. From the theoretical and experimental viewpoints the study of these processes is of interest, since very little is known of the neutrino interaction at intermediate energy in these more complex kinematical domains.

\section{REFERENCES}

1. B. Lehmann-Dronke, A. Schäfer, Phys. Lett. B 52155, (2001).

2. X. Ji, Phys.Rev. D 55 7114, (1997).

3. A. Radyushkin, Phys.Rev. D 56 5524, (1997)

4. C. Corianò, H. Li, C. Savkli. JHEP 9807 008, (1998). 
5. G. Sterman, P. Stoler, Ann. Rev. Nucl. Part. Sci. 47193, (1997).

6. A.V. Belitsky, D. Mueller, L. Niedermeier, A. Schafer Nucl. Phys. B 593 289, (2001).

7. P. Amore, C. Corianò, M. Guzzi, JHEP 0502 038, (2005).

8. C. Corianò, M. Guzzi, Phys.Rev.D 71 053002, (2005).

9. J. Blumlein, D. Robaschik. Nucl.Phys. B 581 449, (2000).

10. A. Psaker, hep-ph/0412321, (2004) 